\title{
Barrier Inhomogeneity and Electrical Properties of InN Nanodots/Si Heterojunction Diodes
}

\author{
Mahesh Kumar, ${ }^{1,2}$ Basanta Roul,, ${ }^{1,2}$ Thirumaleshwara N. Bhat, ${ }^{1}$ Mohana K. Rajpalke, ${ }^{1}$ \\ A. T. Kalghatgi, ${ }^{2}$ and S. B. Krupanidhi ${ }^{1}$ \\ ${ }^{1}$ Materials Research Centre, Indian Institute of Science, Bangalore 560012, India \\ ${ }^{2}$ Central Research Laboratory, Bharat Electronics, Bangalore 560013, India
}

Correspondence should be addressed to S. B. Krupanidhi, sbk@mrc.iisc.ernet.in

Received 25 July 2011; Accepted 28 August 2011

Academic Editor: Zhi Li Xiao

Copyright (C) 2011 Mahesh Kumar et al. This is an open access article distributed under the Creative Commons Attribution License, which permits unrestricted use, distribution, and reproduction in any medium, provided the original work is properly cited.

\begin{abstract}
The electrical transport behavior of $n-n$ indium nitride nanodot-silicon (InN ND-Si) heterostructure Schottky diodes is reported here, which have been fabricated by plasma-assisted molecular beam epitaxy. InN ND structures were grown on a $20 \mathrm{~nm}$ InN buffer layer on Si substrates. These dots were found to be single crystalline and grown along $\left[\begin{array}{llll}0 & 0 & 0 & 1\end{array}\right]$ direction. Temperature-dependent current density-voltage plots $(J-V-T)$ reveal that the ideality factor $(\eta)$ and Schottky barrier height $(\mathrm{SBH})\left(\Phi_{B}\right)$ are temperature dependent. The incorrect values of the Richardson constant $\left(A^{* *}\right)$ produced suggest an inhomogeneous barrier. Descriptions of the experimental results were explained by using two models. First one is barrier height inhomogeneities (BHIs) model, in which considering an effective area of the inhomogeneous contact provided a procedure for a correct determination of $A^{* *}$. The Richardson constant is extracted $\sim 110 \mathrm{~A} \mathrm{~cm}^{-2} \mathrm{~K}^{-2}$ using the BHI model and that is in very good agreement with the theoretical value of $112 \mathrm{~A} \mathrm{~cm}^{-2} \mathrm{~K}^{-2}$. The second model uses Gaussian statistics and by this, mean barrier height $\Phi_{0}$ and $A^{* *}$ were found to be $0.69 \mathrm{eV}$ and $113 \mathrm{~A} \mathrm{~cm}^{-2} \mathrm{~K}^{-2}$, respectively.
\end{abstract}

\section{Introduction}

Group III nitrides represent a material class with promising electronic and optical properties [1]. Among these, InN possesses the lowest effective mass, the highest mobility, narrow band gap $E_{g}$ of $0.7-0.9 \mathrm{eV}$ and the highest saturation velocity $[2,3]$, which make this an attractive material for applications in solar cells, terahertz emitters, and detectors [4-6]. Good quality InN layers are difficult to grow because of the low dissociation temperature of $\mathrm{InN}$ and the lack of an appropriate substrate $[7,8]$. The above constraints lead to the formation of dislocations and strain in the grown epitaxial layers resulting in the degradation of the device performance. Grandal et al. [9] reported that defect- and strain-free InN nanocolumns of very high crystal quality can be grown by molecular beam epitaxy (MBE) with and without buffer layer on silicon substrates. Since silicon is the most sought semiconductor material, it is very important to understand the transport mechanism of $\mathrm{InN}$ nanostructure- based devices and their behavior at different temperatures prior to their adoption in the fabrication of optoelectronic devices. In the present study, InN nanodot (ND) structures were grown on $\mathrm{Si}$ substrates using an $\mathrm{InN}$ buffer layer by plasma-assisted MBE.

The interfaces of the semiconductor heterostructures are important part of semiconductor electronic and optoelectronic devices. One of the most interesting properties of a semiconductor heterostructure interface is its Schottky barrier height $(\mathrm{SBH})\left(\Phi_{B}\right)$, which is a measure of the mismatch of the energy levels for the majority carriers across the interface. Temperature-dependent ideality factor $(\eta)$ and SBH $\left(\Phi_{B}\right)$ and incorrect values of the Richardson constant $\left(A^{* *}\right)$ suggest an inhomogeneous barrier. Two techniques exist to modify the classic Richardson plot to extract the barrier height, taking into account SBH lowering due to an inhomogeneous contact. The first involves extracting the barrier height relevant to $J-V$ analyses, a value referred to as the effective barrier height $\Phi_{\text {eff. }}$ For this technique, Tung [10] 
developed a barrier height inhomogeneities (BHIs) model of such inhomogeneities on the electron transport across the metal semiconductor interface, taking into account the possible presence of a distribution of nanometer-sized "patches" with lower barrier height embedded in a uniform high barrier background. Gammon et al. [11] has been reported that same BHI model can be applied to inhomogeneous heterojunction Schottky contacts. The second technique uses Gaussian statistics to modify the classic Richardson plot to extract the barrier height, taking into account SBH lowering due to an inhomogeneous contact.

In this article, we have studied the electrical transport behavior of $n-n$ InN ND/Si heterostructure Schottky diodes that have been fabricated by plasma-assisted MBE. These electrical measurements showed a dependence on the temperature of the ideality factor $(\eta)$ and the $\operatorname{SBH}\left(\Phi_{B}\right)$ that can be explained by BHI and Gaussian statistics model. The BHI model provided a method for a correct determination of the Richardson constant. Hence, the observed underestimation of the value $A^{* *}$ could be attributed to an effective area, involved in the current transport, which may be significantly lower than the geometric area of the diode.

\section{Experimental Procedure}

The growth system used in this study was a plasma-assisted MBE system equipped with a radio frequency (RF) plasma source. The base pressure in the system was below $1 \times 10^{-10}$ mbar. The $n$-Si $(111)\left(\sim 2 \times 10^{16} \mathrm{~cm}^{-3}\right)$ wafers were first chemically cleaned followed by dipping in 5\% HF to remove the surface oxide and then thermally cleaned at $900^{\circ} \mathrm{C}$ for one hour in ultrahigh vacuum. The deposition of $\mathrm{InN}$ consists of a two-step growth method. The initial low temperature buffer layer of thickness $\sim 20 \mathrm{~nm}$ was deposited at $400^{\circ} \mathrm{C}$. Further, the substrate temperature was raised to $500^{\circ} \mathrm{C}$ to fabricate the NDs. The duration of NDs growth was kept for $60 \mathrm{~min}$. The general set of growth conditions includes indium beam equivalent pressure (BEP), nitrogen flow rate, and rf-plasma power, which were kept at $2.1 \times 10^{-7} \mathrm{mbar}$, $0.5 \mathrm{sccm}$, and $350 \mathrm{~W}$, respectively. The structural evaluation of the as-grown NDs was carried out by the high-resolution X-ray diffraction (HRXRD), field emission scanning electron microscopy (FESEM), and transmission electron microscopy (TEM). The aluminum contacts were fabricated by thermal evaporation. The adequate Ohmic nature of the contacts to InN and Si was verified. The device transport characteristics were studied at various temperatures using the probe station attached with the KEITHLEY-236 source measure unit.

\section{Results and Discussion}

Figure 1 shows $2 \theta-\omega$ XRD pattern of the InN NDs grown on Si (111) substrate. From the figure it can be seen that except the substrate peaks, only (0002) InN diffracted peak at $2 \theta=31.32^{\circ}$ is present, indicating the InN NDs to be highly oriented along the [0001] direction of the wurtzite structures of InN. Figure 2(a) shows a typical FESEM image of InN NDs and illustrates that the as-grown NDs are vertically aligned

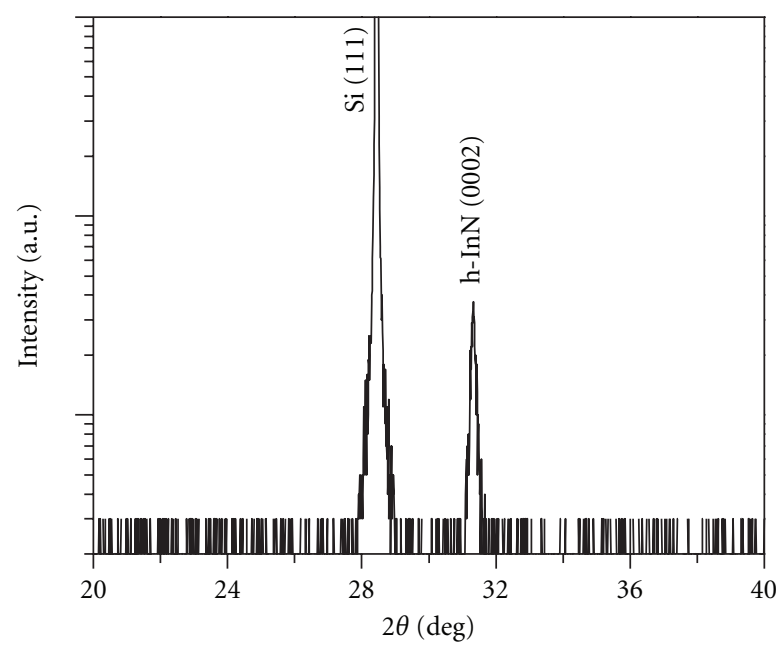

FIgURe 1: HRXRD $2 \theta-\omega$ of InN NDs on $n$-Si (111) substrate.

and uniformly grown over the entire substrate. Figures 2(b)2(d) represent typical TEM micrographs, high-resolution TEM (HRTEM) images, and selected area electron diffraction (SAED) of InN NDs, respectively. The HRTEM shows one of the corner edges of an ND. The interplanar spacing, as observed from the fringe pattern of the HRTEM image, is $0.305 \mathrm{~nm}$, which corresponds to the (1000) lattice spacing of InN. The SAED pattern shows clearly visible bright spots which represent that each ND is single crystalline.

The current density-voltage-temperature $(J-V-T)$ characterization of the Schottky diodes was performed in order to determine the significant parameters ruling the current transport across the InN ND/Si contact, namely, the ideality factor $(\eta)$ and the $\operatorname{SBH}\left(\Phi_{B}\right)$. Figure 3(a) shows a schematic diagram of the device and measurement method. Figure 3(b) shows the semilogarithmic plot of the $J-V$ curves of the InN ND/Si diodes, which were acquired in the temperature range of $120-450 \mathrm{~K}$. An excellent rectifying behavior was observed at lower temperatures, but at high temperatures, a deterioration was observed in the rectifying nature, which may be due to thermally generated carrier tunneling. It is very clear from the $J-V-T$ curve that at fixed bias, the forward current increases with increasing temperature. This indicates that the current is induced by the thermionic emission (TE). The values of $\operatorname{SBH}\left(\Phi_{B}\right)$ and the ideality factor $(\eta)$ for the junction were calculated as a function of measuring temperature by fitting a line in the linear region of the forward $J-V$ curves using the TE equation $[12,13]$ :

$$
J=J_{S}\left\{\exp \left(\frac{q V}{\eta k T}\right)-1\right\},
$$

where $J_{S}$ is the saturation current density expressed by

$$
J_{S}=\frac{I_{S}}{S}=A^{* *} T^{2} \exp \left(-\frac{q \Phi_{B}}{k T}\right) .
$$

Here, $S$ is the area of the diode, $A^{* *}$ is the effective Richardson constant (112 $\mathrm{A} \mathrm{cm}^{-2} \mathrm{~K}^{-2}$ for $n$-type Si) [14], $k$ is the Boltzmann's constant, $q$ is the electron charge, and $T$ is the measure temperature. 

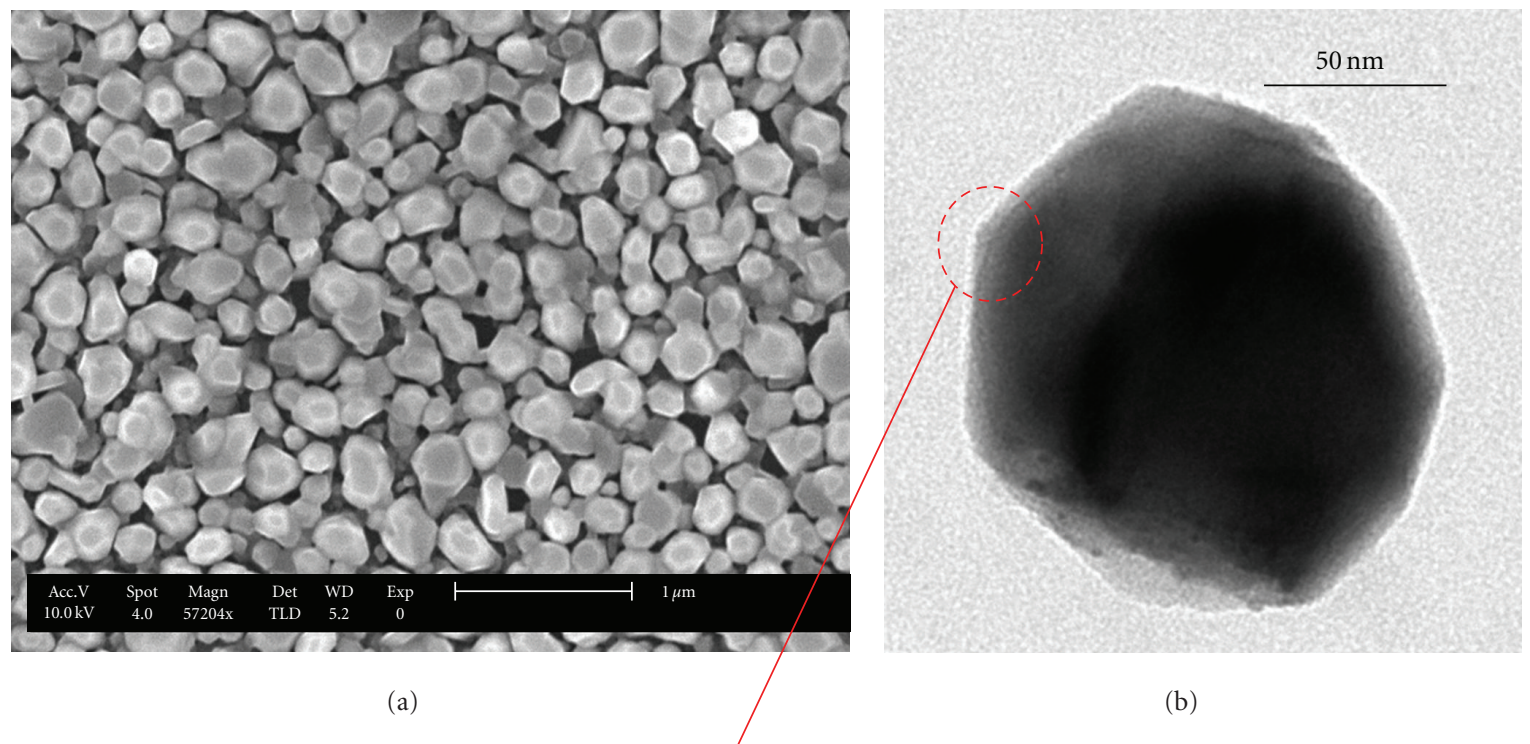

(b)

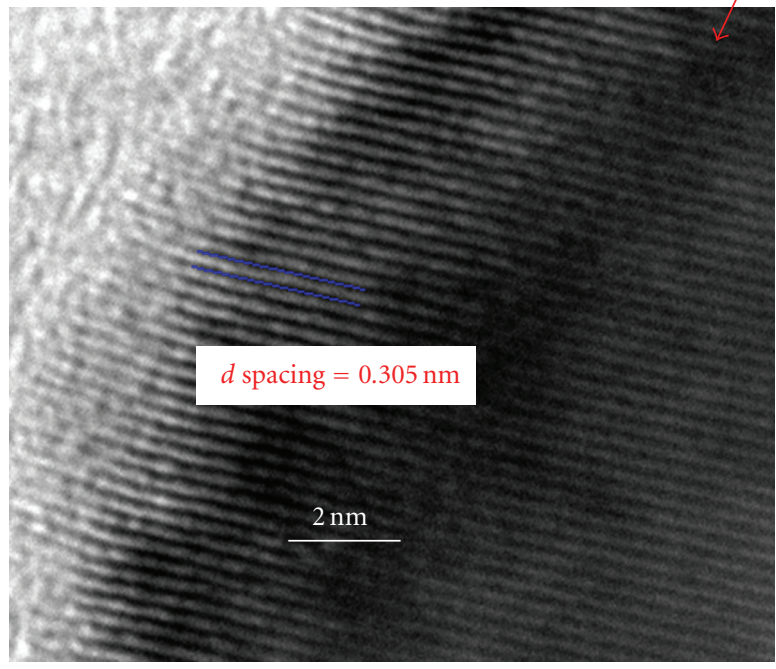

(c)

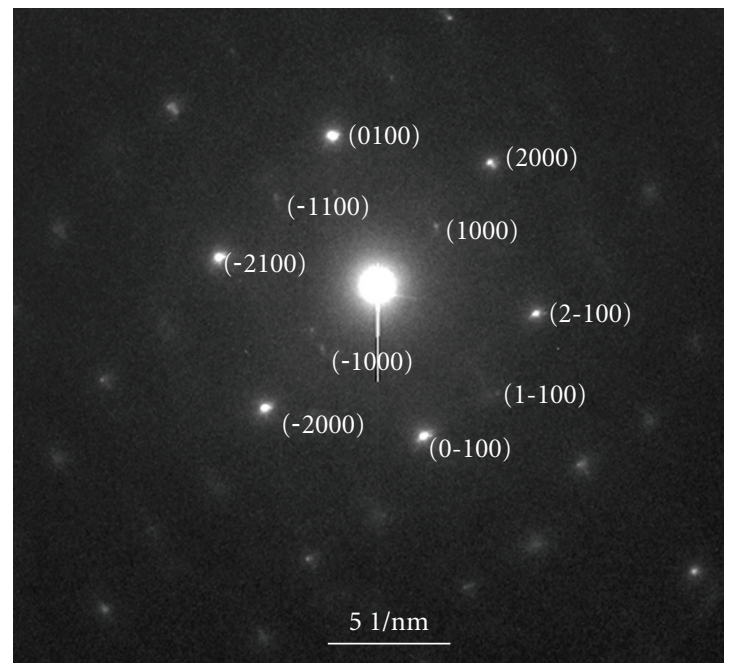

(d)

Figure 2: (a) FESEM image of the InN NDs, (b) typical TEM image of a ND, (c) HRTEM of the corner edge of a ND, (d) SAED pattern taken along the [0001] direction.

Figure 4 shows the ideality factor $(\eta)$ and the $\mathrm{SBH}\left(\Phi_{B}\right)$ of InN ND/Si Schottky diodes as function of temperature, which have been extracted from the forward bias $J-V$ characteristics. It may be clearly seen that these parameters are both temperature dependent. In particular, by decreasing temperatures the ideality factor deviates from the unity and SBH $\left(\Phi_{B}\right)$ decreases from the value of $0.68 \mathrm{eV}$ at $450 \mathrm{~K}$ to $0.48 \mathrm{eV}$ at $120 \mathrm{~K}$. The deviation from the ideality observed at lower temperatures suggests the presence of an inhomogeneous barrier. Further, to justify the application of the BHI model to our data, the temperature dependence of the ideality factor $\eta$ was reported in a plot of $\eta k T$ versus $k T$ and is shown in Figure 5, in which the straight line shows an ideal behavior of a Schottky contact $(\eta=1)$. The experimental data could be fitted by a straight line, which is parallel to that of the ideal Schottky contact behavior and the ideality factor $(\eta)$ can be expressed in the form $\eta \sim 1+T_{0} / T$, with $T_{0}=31 \pm 3 \mathrm{~K}$ in present case, as determined by the fit parameters. This behavior is referred as the " $T_{0}$ anomaly" [15]. Such parametric dependence is typical of a real Schottky contact with a distribution of barrier inhomogeneities [16].

Plotting the $\mathrm{SBH}\left(\Phi_{B}\right)$ against their respective ideality factors as shown in Figure 6 displays the linear correlation between the two. Extrapolating a linear fit of the data to $\eta=1$ reveals the average barrier height $\Phi_{B}^{0}[17]$. By the extrapolation of $\operatorname{SBH}\left(\Phi_{B}\right)$ at $\eta=1$ of the experimental data reported in Figure 6, a value of $\Phi_{B}^{0}=0.75 \mathrm{eV}$ was determined. Another technique used to extract the barrier height is via a Richardson plot. The values of the saturation current density $\left(J_{s}=I_{s} / S\right)$ were determined from the extrapolation at $V=0$ of the linear fit of the $I-V$ curves in the range $280-450 \mathrm{~K}$, that is, where the deviation from the ideality is small. The conventional Richardson's plot of 


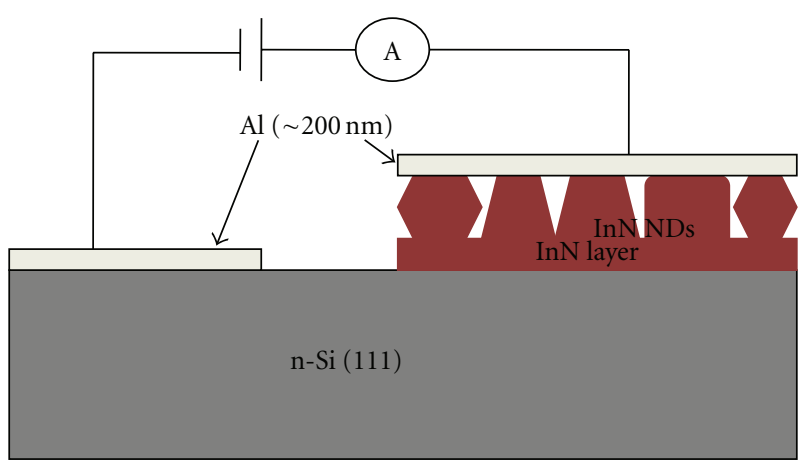

(a)

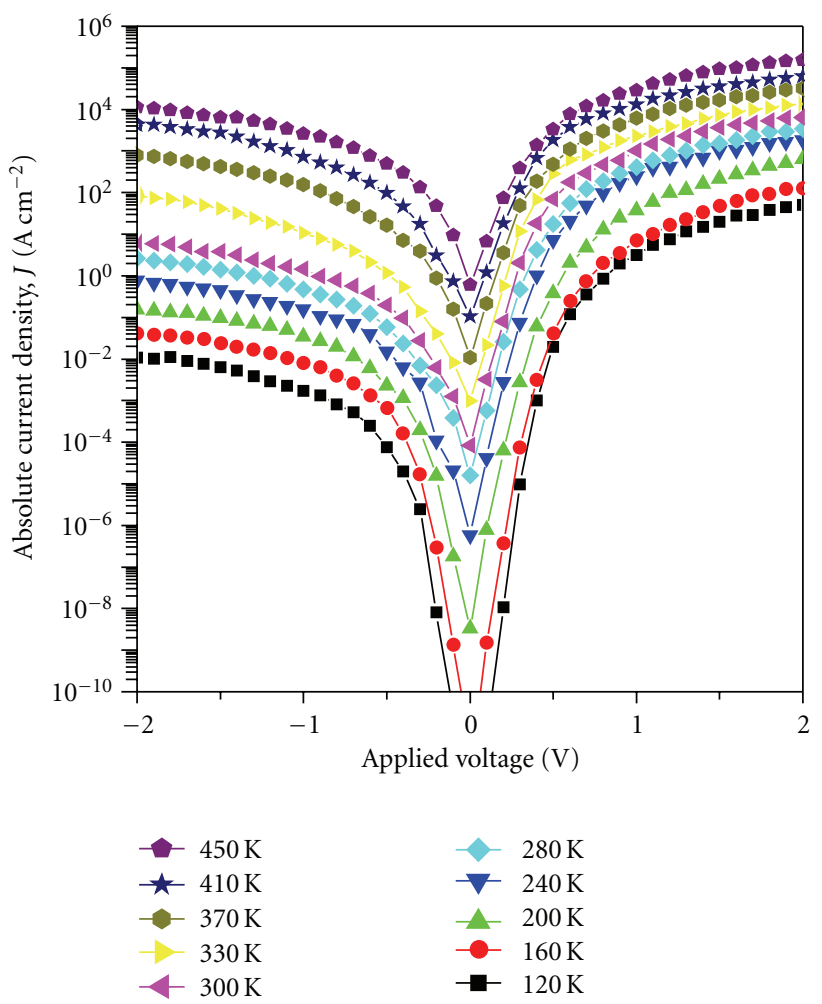

(b)

FIgure 3: (a) A schematic diagram of the device and measurement method. (b) Current density versus voltage plots of the InN ND/Si diode at different temperatures.

$\ln \left(J_{s} / T^{2}\right)$ versus $1 / k T$ was obtained and is shown in Figure 7. From the linear fit to the plot, the Richardson constant $\left(A^{* *}\right)$ and effective Schottky barrier height $\left(\Phi_{\text {eff }}\right)$ were calculated to be $\sim 16 \mathrm{~A} \mathrm{~cm}^{-2} \mathrm{~K}^{-2}$ and $0.57 \mathrm{eV}$, respectively. The value of $A^{* *}$ obtained from the conventional Richardson plot is more than a factor of seven lower with respect to the theoretical value of $112 \mathrm{~A} \mathrm{~cm}^{-2} \mathrm{~K}^{-2}$ for $n$-type Si. Also, the value of effective barrier height $\Phi_{\text {eff }}$ is less than the average barrier height $\Phi_{B}^{0}$, suggesting the formation of an inhomogeneous $\mathrm{SBH}$ at the interface.

The expression of the current flowing through the InN $\mathrm{ND} / \mathrm{Si}$ Schottky diodes can be obtained by adding the current through the low barrier patches to the current which passes

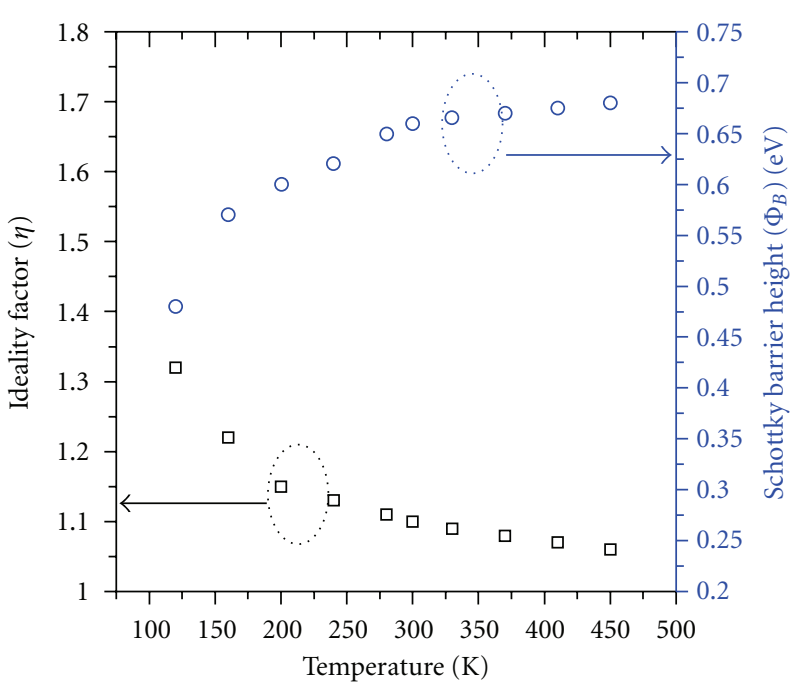

FIGURE 4: The temperature dependence of the ideality factor $(\eta)$ and $\mathrm{SBH}\left(\Phi_{B}\right)$ from an InN ND/Si heterojunction diode.

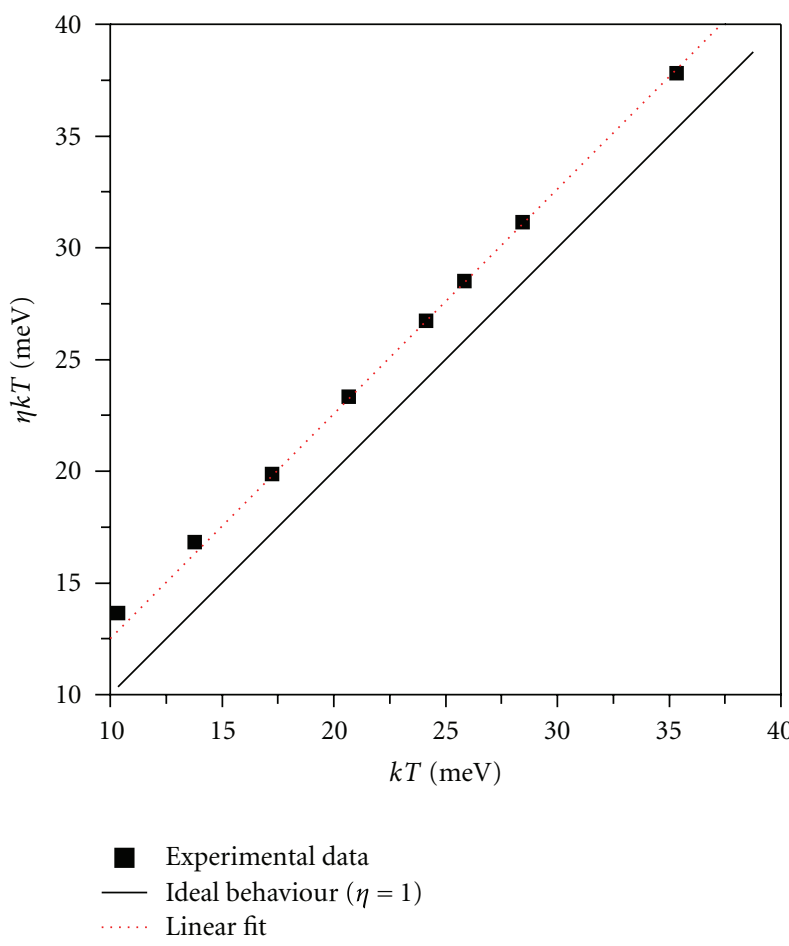

FIgure 5: Plot of $n k T$ as a function of $k T$ showing the $T_{0}$ anomaly $\eta \sim 1+T_{0} / T$. The linear fit of the data is also reported, from which a value of $T_{0}=31 \mathrm{~K}$ was calculated. For reference, the straight line of the ideal behavior $n=1$ is also reported.

through the surrounding homogeneous regions. However, it is often reasonably assumed that the $I-V$ characteristic is dominated by the current flow through the low SBH patches. Considering the TE equations ( $(1)$ and (2)), the area $S$ can be replaced with the product $N S_{\text {eff, }}$, which represents the total area of a contact made up of a low SBH. Individually, $N$ is the number of patches in the area $S$ and $S_{\text {eff }}$ is the area of 


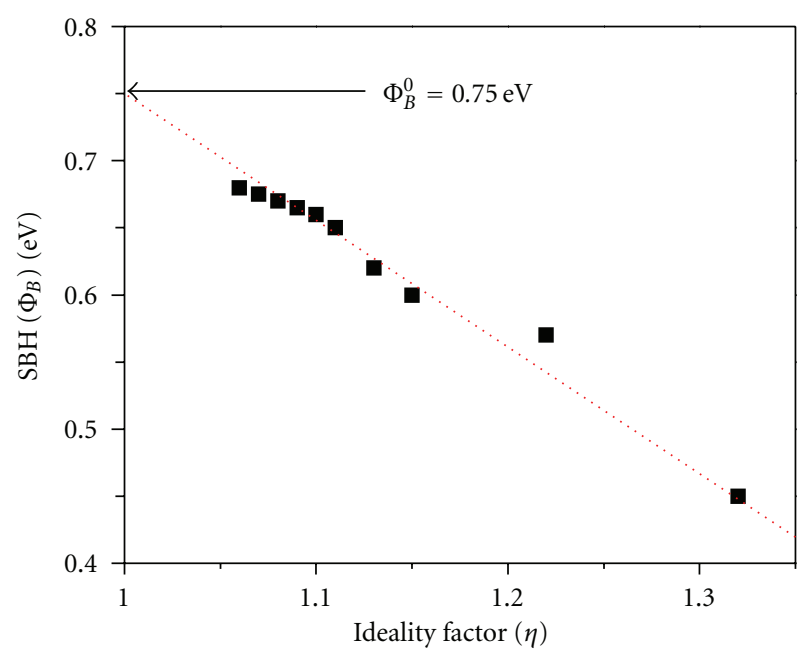

FIGURE 6: The barrier height plotted against the ideality factor, the extrapolation to $n=1$ via a linear fit gave the average barrier height $\Phi_{B}^{0}=0.75 \mathrm{eV}$.

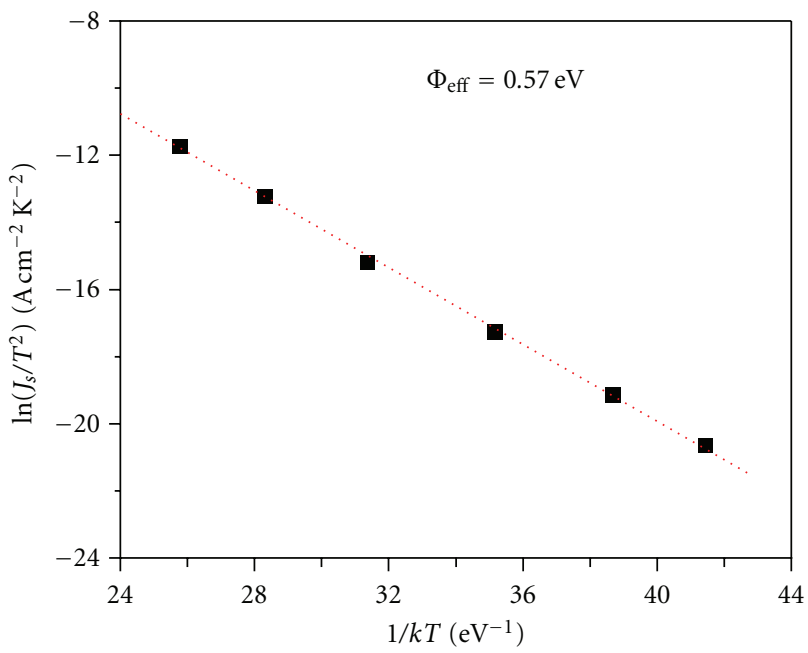

FIgURe 7: Richardson plot $\ln \left(J_{s} / T^{2}\right)$ versus $1 / k T$. From the slope of the linear fit of the data, an effective $\mathrm{SBH}$ of $0.57 \mathrm{eV}$ was determined.

a single patch of a low SBH. Equation (1) can be rewritten substituting in the product $N S_{\text {eff }}$

$$
I_{\text {eff }}=N S_{\text {eff }} A^{* *} T^{2} \exp \left(-\frac{q \Phi_{\text {eff }}}{k T}\right)\left\{\exp \left(\frac{q V}{\eta k T}\right)-1\right\}
$$

where the SBH modeling parameter $\Phi_{\text {eff }}$ replaces $\Phi_{B}$. The effective area $S_{\text {eff }}$ of the low SBH patch can be expressed as

$$
S_{\mathrm{eff}}=\frac{4 \pi \eta^{*} \cdot k T}{9 q V_{\mathrm{bb}}}\left(\Phi_{B}^{0}-\Phi_{\mathrm{eff}}\right),
$$

where $V_{\mathrm{bb}}$ is the band bending at the InN ND/Si interface and $\eta^{*}=\varepsilon_{s} / q \mathrm{~N}_{\mathrm{D}}$.

The experimental $I-V$ curves were fitted by using (3) with the number of patches $N$ as free parameter. A value of $N=6 \times 10^{7}$ gave a good fit of the experimental data in

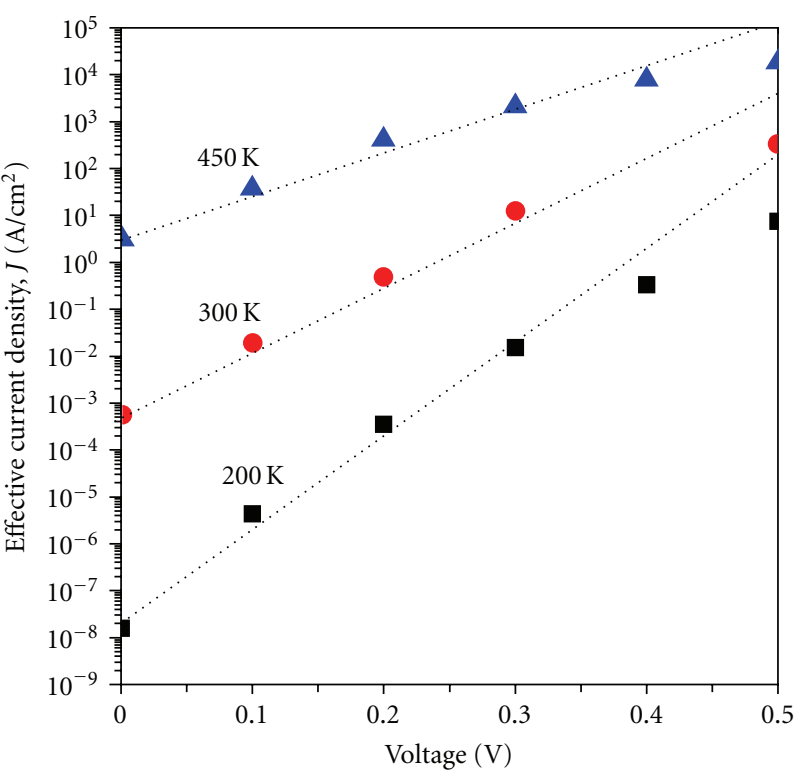

FIGURE 8: Experimental $J-V$ plots at the temperatures of $450 \mathrm{~K}$, $300 \mathrm{~K}$, and $200 \mathrm{~K}$, and calculated curves (dotted lines) obtained by applying the BHI model to the InN ND/Si contact.

the investigated range of temperatures. The product $N S_{\text {eff }}$ represents the total effective area contributing to the current transport. Although $N$ is independent of temperature, as it gives the number of patches in the contact, the product $N S_{\text {eff }}$ exhibits temperature dependence. Figure 8 shows that selecting the correct balance of the variables $N, \Phi_{\text {eff }}$, and ideality factor $(\eta)$ provides a very good approximation to the linear fits of the experimental data. The values of $\eta$ used were those extracted from each individual $J-V$ plot, as was illustrated in Figure 4 . The values of $\Phi_{\text {eff }}$ and $N$ were arrived at by using a modified Richardson plot. The product $N S_{\text {eff }}$ is temperature dependent, in order to eliminate the temperature dependence on the SBH within a Richardson plot, the total area dominated by the low $\mathrm{SBH}$ patches $\left(N S_{\text {eff }}\right)$ is taken into consideration within the Richardson plot is (3) rearranged to

$$
\ln \left(\frac{I_{\text {eff,S }}}{N S_{\text {eff }} \mathrm{T}^{2}}\right)=\ln \left(A^{* *}\right)-\frac{q \Phi_{\text {eff }}}{k T} .
$$

Figure 9 shows the modified Richardson's plot. From the slope of the straight line fitting the data, the value of $\Phi_{\text {eff }}$ was obtained $\sim 0.57 \mathrm{eV}$, while from the intercept, a value of the Richardson constant of $A^{* *}=110 \mathrm{~A} \mathrm{~cm}^{-2} \mathrm{~K}^{-2}$ was determined and this is very close to the theoretical value of $112 \mathrm{~A} \mathrm{~cm}^{-2} \mathrm{~K}^{-2}$. The product of $N S_{\text {eff }}$ was found $8.27 \times$ $10^{-5} \mathrm{~cm}^{2}$ at room temperature, represented $11 \%$ of the total area.

The other technique used the Gaussian statistics [1820] to modify the classic Richardson plot to extract the barrier height, taking into account SBH lowering due to an inhomogeneous contact. This technique uses Gaussian statistics to relate experimental values of $\mathrm{SBH}$ extracted from $I-V$ analysis $\Phi_{B}$, back to the mean SBH $\Phi_{0}$. The amount 


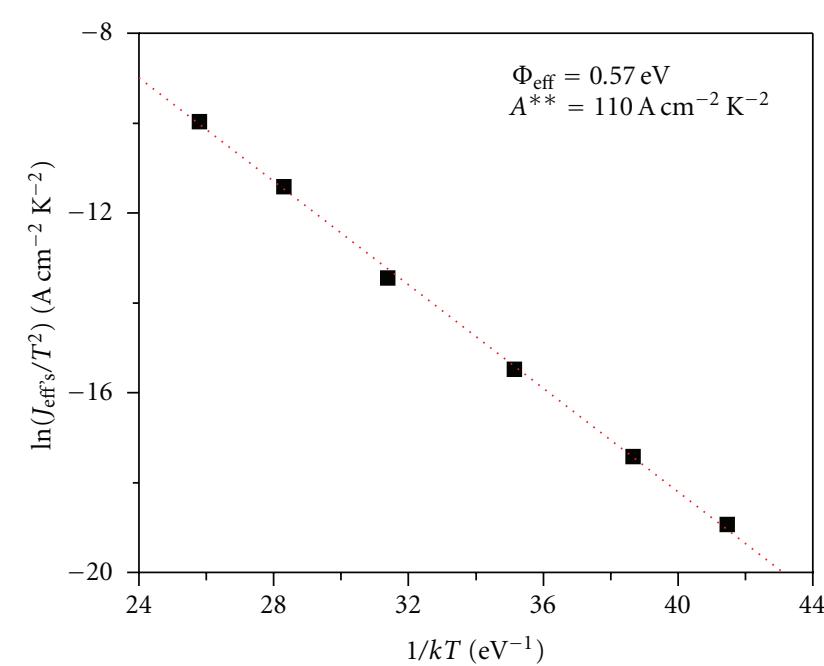

FIGURE 9: The modified Richardson plots for the extraction of the effective SBH ( $\left.\Phi_{\text {eff }}\right)$ and an effective barrier height of $0.57 \mathrm{eV}$ were extracted from $\ln \left(J_{\text {eff, } s} / T^{2}\right)$ versus $1 / k T$.

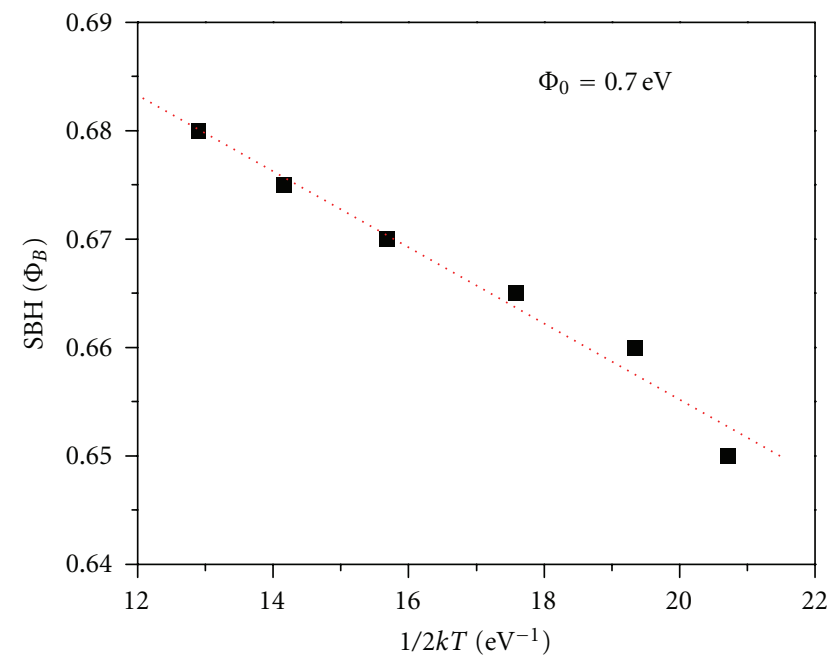

FIGURE 10: The barrier heights extracted from $I-V$ analysis plotted against the $1 / 2 k T$.

of patches $(d n)$ that will have SBH values falling between $\Phi_{0}$ and the value of SBH measured from the individual $I-V$ curves $\Phi_{B}$ have a Gaussian distribution given by

$$
d n=\frac{N}{\sigma \sqrt{2 \pi}} \exp \left[-\frac{\left(\Phi_{0}-\Phi_{B}\right)^{2}}{2 \sigma^{2}}\right] d \Phi_{0}
$$

where $\sigma$ is the standard deviation of the distribution and $N$ is the total number of patches in the area $S$. Solving (1), (2), and (6), the total forward current can be given by [11]

$$
I=S A^{* *} T^{2} \exp \left(-\frac{q \Phi_{B}}{k T}+0.5 \frac{q^{2} \sigma^{2}}{(k T)^{2}}\right)\left\{\exp \left(\frac{q V}{\eta k T}\right)-1\right\} .
$$

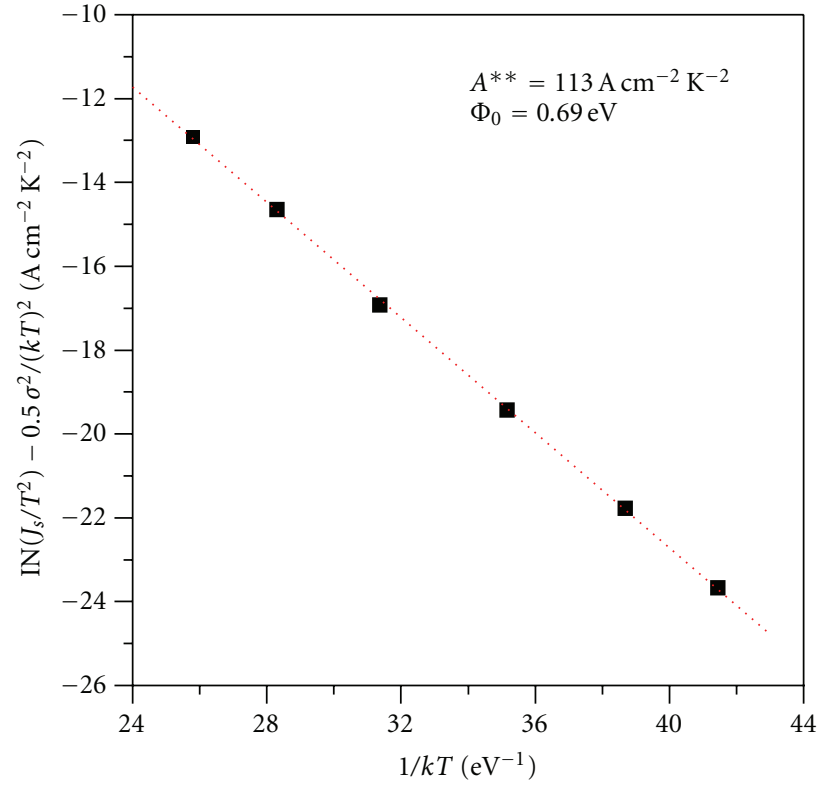

FIGURE 11: The modified Richardson plots for the extraction of mean SBH $\Phi_{0}$ and mean barrier height of $0.69 \mathrm{eV}$ were extracted $\ln \left(J_{s} / T^{2}\right)-0.5 \beta^{2} \sigma^{2}$ versus $1 / k T$.

The TE equation for current over the barrier $\Phi_{0}$ is

$$
\mathrm{I}=S A^{* *} T^{2} \exp \left(-\frac{q \Phi_{0}}{k T}\right)\left\{\exp \left(\frac{q V}{\eta k T}\right)-1\right\} .
$$

Combining (7) and (8) and rearranging

$$
\Phi_{B}=\Phi_{0}-\frac{\mathrm{q} \sigma^{2}}{2 \mathrm{kT}},
$$

this allows the values of SBH measured from the $I-V$ analysis to be plotted against the inverse thermal energy, to extract $\sigma$ and $\Phi_{0}$. This is shown in Figure 10 where $\sigma$ and $\Phi_{0}$ were found to be $0.0593 \mathrm{eV}$ and $0.70 \mathrm{eV}$, respectively. Verification of this value can be carried out using a Richardson plot, and rearranging (7) and (9):

$$
\ln \left(\frac{J_{S}}{T^{2}}\right)-\frac{1}{2}\left(\frac{q \sigma}{k T}\right)^{2}=\ln \left(A^{* *}\right)-\frac{q \Phi_{0}}{k T} .
$$

Figure 11 shows the resulting Richardson plot where $\Phi_{0}$ was found to be $0.69 \mathrm{eV}$ and $A^{* *}$ was $113 \mathrm{~A} \mathrm{~cm}^{-2} \mathrm{~K}^{-2}$.

\section{Conclusions}

We have demonstrated the electrical transport behavior of $n$-InN ND/Si heterostructure Schottky diodes that have been fabricated by plasma-assisted MBE. Singlecrystalline wurtzite structures of $\mathrm{InN} \mathrm{NDs}$ are verified by the X-ray diffraction and transmission electron microscopy. Temperature-dependent current-voltage plots $(I-V-T)$ reveal that the ideality factor $(\eta)$ and Schottky barrier height $(\mathrm{SBH})\left(\Phi_{\mathrm{B}}\right)$ are temperature dependent and that incorrect values of the Richardson constant $\left(A^{* *}\right)$ 
are being produced, suggesting an inhomogeneous barrier. Descriptions of the experimental results were explained by using BHI and Gaussian statistics models. The Richardson constant was extracted to be $\sim 110 \mathrm{~A} \mathrm{~cm}^{-2} \mathrm{~K}^{-2}$ using the BHI model and that is in very good agreement with the theoretical value of $112 \mathrm{~A} \mathrm{~cm}^{-2} \mathrm{~K}^{-2}$. The second model uses Gaussian statistics and by this, mean barrier height $\Phi_{0}$ and $A^{* *}$ were found to be $0.69 \mathrm{eV}$ and $113 \mathrm{~A} \mathrm{~cm}^{-2} \mathrm{~K}^{-2}$, respectively.

\section{References}

[1] S. Nakamura, "The roles of structural imperfections in InGaN-based blue light- emitting diodes and laser diodes," Science, vol. 281, no. 5379, pp. 956-961, 1998.

[2] J. Wu, W. Walukiewicz, K. M. Yu et al., "Unusual properties of the fundamental band gap of InN," Applied Physics Letters, vol. 80, no. 21, p. 3967, 2002.

[3] M. Kumar, B. Roul, T. N. Bhat et al., "Kinetics of selfassembled InN quantum dots grown on Si (111) by plasmaassisted MBE," Journal of Nanoparticle Research, vol. 13, p. 1281, 2011.

[4] H. C. Yang, P. F. Kuo, T. Y. Lin et al., " Mechanism of luminescence in InGaN/GaN multiple quantum wells," Applied Physics Letters, vol. 76, no. 25, p. 3712, 2000.

[5] E. Bellotti, B. K. Doshi, K. F. Brennan, J. D. Albrecht, and P. P. Ruden, "Ensemble Monte Carlo study of electron transport in wurtzite InN," Journal of Applied Physics, vol. 85, no. 2, pp. 916-923, 1999.

[6] C. Y. Chang, G. C. Chi, W. M. Wang et al., "Transport properties of InN nanowires," Applied Physics Letters, vol. 87, no. 9, Article ID 093112, 2005.

[7] Q. Guo, O. Kato, and A. Yoshida, "Thermal stability of indium nitride single crystal films," Journal of Applied Physics, vol. 73, no. 11, pp. 7969-7971, 1993.

[8] K. Wang and R. R. Reeber, "Thermal expansion and elastic properties of InN," Applied Physics Letters, vol. 79, no. 11, pp. 1602-1604, 2001.

[9] J. Grandal, M. A. Sánchez-García, E. Calleja, E. Luna, and A. Trampert, "Accommodation mechanism of InN nanocolumns grown on $\mathrm{Si}(111)$ substrates by molecular beam epitaxy," Applied Physics Letters, vol. 91, no. 2, Article ID 021902, 2007.

[10] R. T. Tung, "Electron transport at metal-semiconductor interfaces: general theory," Physical Review B, vol. 45, no. 23, pp. 13509-13523, 1992.

[11] P. M. Gammon, A. Pérez-Tomás, V. A. Shah et al., "Analysis of inhomogeneous $\mathrm{Ge} / \mathrm{SiC}$ heterojunction diodes," Journal of Applied Physics, vol. 106, no. 9, Article ID 093708, 2009.

[12] L. Wang, M. I. Nathan, T. H. Lim, M. A. Khan, and Q. Chen, "High barrier height GaN Schottky diodes: Pt/GaN and Pd/GaN," Applied Physics Letters, vol. 68, no. 9, pp. 1267-1269, 1996.

[13] H. Morkoc, Handbook of Nitride Semiconductors and Devices, Wiley-VCH, New York, NY, USA, 2008.

[14] C. Hayzelden and J. L. Batstone, "Silicide formation and silicide-mediated crystallization of nickel-implanted amorphous silicon thin films," Journal of Applied Physics, vol. 73, no. 12 , pp. 8279-8289, 1993.

[15] F. Roccaforte, F. La Via, V. Raineri, R. Pierobon, and E. Zanoni, "Richardson's constant in inhomogeneous silicon carbide Schottky contacts," Journal of Applied Physics, vol. 93, no. 11, pp. 9137-9144, 2003.

[16] B. Abay, G. Cankaya, H. S. Guder, H. Efeoglu, and Y. K. Yogurtcu, "Barrier characteristics of Cd/p-GaTe Schottky diodes based on I-V-T measurements," Semiconductor Science and Technology, vol. 18, no. 2, pp. 75-81, 2003.

[17] R. F. Schmitsdorf, T. U. Kampen, and W. Mönch, "Explanation of the linear correlation between barrier heights and ideality factors of real metal-semiconductor contacts by laterally nonuniform Schottky barriers," Journal of Vacuum Science and Technology B, vol. 15, no. 4, pp. 1221-1226, 1997.

[18] Y. P. Song, R. L. Van Meirhaeghe, W. H. Laflère, and F. Cardon, "On the difference in apparent barrier height as obtained from capacitance-voltage and current-voltagetemperature measurements on $\mathrm{Al} / \mathrm{p}$-InP Schottky barriers," Solid State Electronics, vol. 29, no. 6, pp. 633-638, 1986.

[19] J. H. Werner and H. H. Güttler, "Barrier inhomogeneities at Schottky contacts," Journal of Applied Physics, vol. 69, no. 3, pp. 1522-1533, 1991.

[20] F. E. Jones, B. P. Wood, J. A. Myers, C. Daniels-Hafer, and M. C. Lonergan, "Current transport and the role of barrier inhomogeneities at the high barrier n-InP - poly(pyrrole) interface," Journal of Applied Physics, vol. 86, no. 11, pp. 64316441, 1999. 

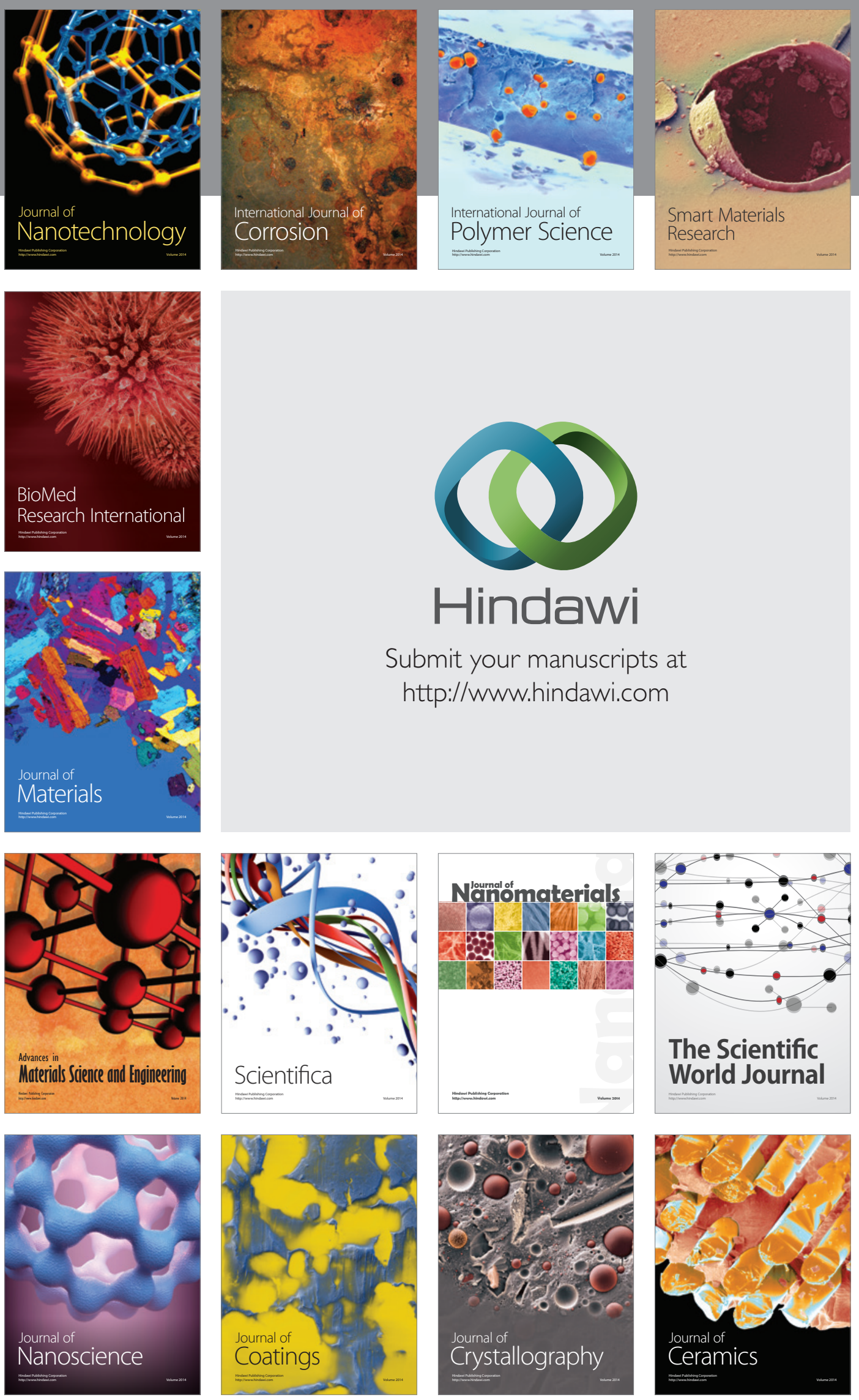

The Scientific World Journal

Submit your manuscripts at

http://www.hindawi.com

\section{World Journal}

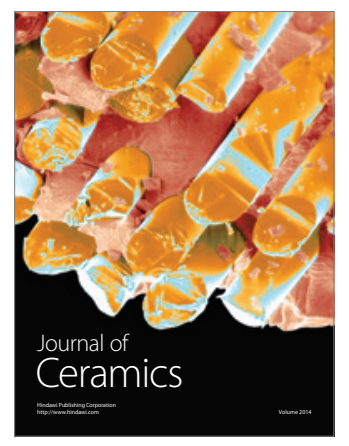

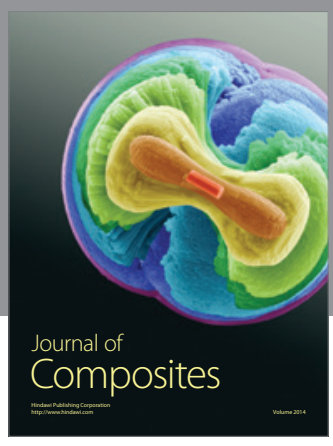
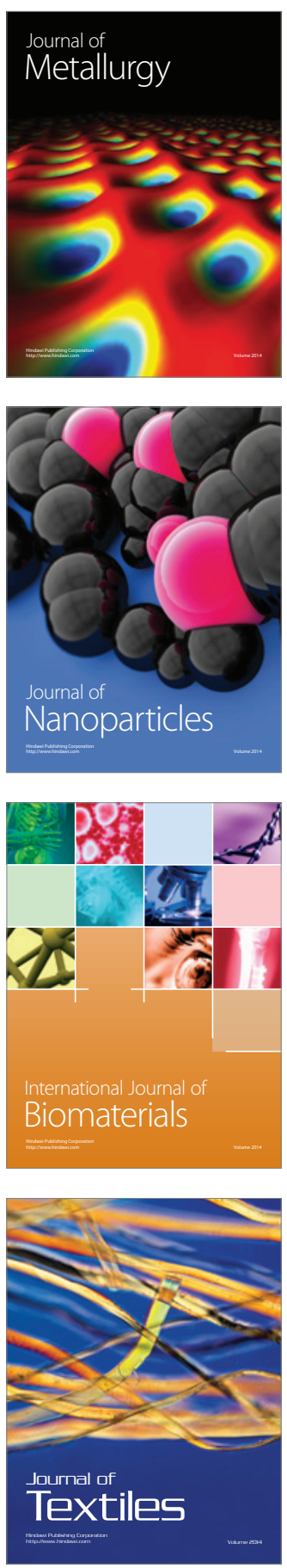